\title{
Correlation Analysis of Nodular Sonographic Parameters with Cervical Lymph Node Metastasis in Papillary Thyroid Carcinoma
}

\section{Liuhua Zhou}

Zhejiang Chinese Medical University

\section{Qiaodan Zhu}

Zhejiang Chinese Medical University

Jincao Yao

Cancer Hospital of the University of Chinese Academy of Sciences (Zhejiang Cancer Hospital)

\section{Chen Yang}

Cancer Hospital of the University of Chinese Academy of Sciences (Zhejiang Cancer Hospital)

Dong Xu ( $\nabla$ xudong@zjcc.org.cn )

Cancer Hospital of the University of Chinese Academy of Sciences (Zhejiang Cancer Hospital)

\section{Research Article}

Keywords: Ultrasonography, Thyroid Cancer, Papillary, Lymphatic metastasis

Posted Date: September 20th, 2021

DOI: https://doi.org/10.21203/rs.3.rs-863723/v1

License: (9) This work is licensed under a Creative Commons Attribution 4.0 International License. Read Full License 


\section{Abstract \\ Objective}

Papillary thyroid carcinoma (PTC) is the most common thyroid carcinoma, and is prone to cervical lymph node metastasis (CLNM). We aim to analyze the correlation between clinical information, ultrasonic parameters of PTC and CLNM.

\section{Methods}

1335 patients who had pathologically confirmed unifocal PTC were enrolled. Univariate and multivariate logistic analysis were performed to predict CLNM in PTC patients. The receiver operating characteristic (ROC) curve was used to evaluate the diagnostic performance.

\section{Results}

Univariate analysis showed that gender, age, maximum tumor diameter and volume, cross-sectional and longitudinal aspect ratio were related to CLNM $(P<0.05)$. Multivariate logistic analysis showed that gender, age, maximum tumor diameter and volume were independent correlative factors, cross-sectional aspect ratio had significant difference for PTC patients excluding PTMC to predict CLNM. The area under the curve (AUC) of the maximum tumor diameter and volume was 0.738 and 0.733 , respectively. Maximum tumor diameter and volume, and cross-sectional and longitudinal aspect ratio were statistically significant following analysis of variance $(P<0.05)$.

\section{Conclusions}

Younger age, male, and larger tumor were high risk factors for CLNM in patients with unifocal PTC. Crosssectional aspect ratio had a more effective predictive value for CLNM in patients with larger thyroid tumors.

\section{Introduction}

Papillary thyroid carcinoma (PTC) is the most common pathological type of thyroid carcinoma, accounting for approximately 70-80\% [1-2]. Early and timely PTC surgery results in better prognosis; however, PTC is prone to developing cervical lymph node metastasis (CLNM), and there is a risk of postoperative recurrence and distant metastasis [3]. Although intraoperative lymph node dissection can effectively reduce the residual tumor and recurrence, complications such as recurrent laryngeal nerve injury and hypoparathyroidism can occur. Whether to perform preventive dissection of central neck lymph nodes is still a hot topic of debate among scholars [4]. Therefore, an effective method to increase the detection rate of metastatic cervical lymph nodes before surgery is required. 
Ultrasonography (US) is currently the primary method for diagnosing thyroid lesions, but it has some limitations in the detection of CLNM, especially for central lymph nodes metastasis, ultrasonography has the detection rate of $18.8 \%-31 \%$ [5-6]. US can be affected by interference due to underlying thyroid diseases, surrounding tissues and is dependent on physician experience. Therefore, the identification of useful clues when performing routine thyroid scanning is essential to improve the detection rate of CLNM.

Based on previous studies, the inclusion criteria for samples were multiplex, multifocal and the aspect ratio (other than cross-sectional or longitudinal), and there was a lack of clear conclusions on tumor volume [7-9]. The present study was based on a stratification which included basic clinical information and US measurements for unifocal PTC, in order to analyze the correlative factors of CLNM. The parameters included were easy to access following basic training in China, especially for thyroid physical examination, and could be the first line of defense to predict the risk of lymph node metastasis before surgery when we perform routine thyroid and cervical lymph node ultrasonic screening.

\section{Materials And Methods}

\section{Patients}

A total of 3570 patients who underwent thyroidectomy in our hospital from July 2014 to September 2018 were enrolled. The inclusion criteria were as follows: 1 . thyroidectomy performed for the first time; 2 . enhanced CT scan of the neck and thorax to assess cervical lymph node and pulmonary metastasis, and US of the thyroid and neck before surgery; 3 . PTC was confirmed by biopsy before surgery. The exclusion criteria were as follows: 1 . lung or other distant metastasis; 2. postoperative pathology diagnosed multifocal PTC. In total, 1335 patients with 1335 lesions were included in the study, and their clinical data and ultrasonic measurements were retrospectively analyzed. There were 299 males and 1036 females, aged 12 to 84 years, with an average age of $45.3 \pm 11.8$ years; the maximum tumor diameter was 3.5 to $64.7 \mathrm{~mm}$, with an average diameter of $10.3 \pm 7.9 \mathrm{~mm}$. Unilateral thyroid lobe plus isthmus excision or total thyroidectomy were performed in these patients. If lateral lymph node metastasis was suspected following a comprehensive preoperative evaluation, and confirmed by biopsy, lateral lymph node dissection was performed [10]. All patients underwent preventive central lymph node dissection [11-12]. Irrespective of whether the dissected lymph node metastasized, metastatic lymph nodes were confirmed by pathology. This study was approved by the Ethics Committee of the Cancer Hospital of the University of Chinese Academy of Sciences (Zhejiang Cancer Hospital), and all enrolled patients signed an informed consent form.

\section{Instruments}

GE Logiq E9 ultrasonographic instrument (General Electric Healthcare, Milwaukee, WI, USA) with a highresolution linear probe (ML6-15) and Philips iU22 ultrasonographic instrument (Royal Dutch Philips Electronics, Amsterdam, Noord-Holland, Netherlands) with a high frequency linear probe (L12-5) were used for the examination. 


\section{Protocol}

Patients were maintained in the supine position with the neck hyper-extended, and then the thyroid and both sides of the neck were scanned in multi-sections. The length, depth, width, location, composition, echogenicity, shape, margin and echogenic foci of the tumor were recorded and evaluated from workstations. The ultrasonic images were obtained by the same two professional physicians with more than 10 years' experience, both were board-certified physicians with training and experience in thyroid US. The ultrasonic images and reports were analyzed in a blinded manner by two ultrasound specialists (with more than 10 years' experience) independently. The imaging data were compared with the pathological results from neck dissections. In cases of discordance, experienced sonologists (with more than 20 years' experience) in thyroid US reviewed the images and made the final decision.

Clinical information and US measurements were collected. Clinical information included gender and age. An age threshold of 55 years was used for analysis according to the 8th edition of the United States Joint Committee on Cancer, as the diagnostic age of the TNM staging system for thyroid cancer was 55 years [13]. US measurements included the maximum tumor diameter, tumor volume $(\mathrm{V}=$ $0.523 *$ length*width*depth), cross-sectional aspect ratio and longitudinal aspect ratio.

The three diameters of the tumour were stated precisely as the following, we did a longitudinal scan of thyroid, selected the maximum section of the nodule, measured the maximum long diameter, it was length, then measured vertical diameter of long diameter, it was depth. Transversal scan of thyroid was executed, selected the maximum section of the nodule, measured the maximum diameter from left to right, it was width. Cross-sectional aspect ratio $=$ depth/width and longitudinal aspect ratio $=$ depth/length.

\section{Statistical analysis}

The obtained data were statistically analyzed by SPSS 20.0 software. Continuous quantitative data were expressed as the mean \pm standard deviation (SD). Data-counting were described statistically by the number of cases and rates. Chi-square test and independent-sample $T$ test were used for univariate analysis. A multivariate analysis using binary logistic regression analysis was adopted if analysis index $p<0.05$ in the univariate analysis.

Odds ratios (ORs) with $95 \%$ confidence intervals (Cls) were calculated and receiver operating characteristic curves (ROC) were analyzed for factors with significance on binary linear regression analysis. Analysis of variance (ANOVA) was used for the positive group. The potential errors due to multiple comparisons for secondary and sub-group were handled by adjusting significance threshold. $\mathrm{P}<$ 0.05 was considered statistically significant.

\section{Results}


According to the inclusion and exclusion criteria, a total of 1335 patients were included in this study. There were 874 cases of PTMC and 461 cases of PTC patients excluding PTMC, 432 cases were in positive group (32.4\%), PTMC patients were 181 cases, PTC excluding PTMC were 251 cases. Among the positive group, $285(21.4 \%)$ with only central lymph node metastasis, $54(4.0 \%)$ with only lateral lymph node metastasis, and 93 (7.0\%) with metastasis both in central and lateral lymph node) were included. There were 903 cases included in negative group (67.6\%), PTMC patients were 693 cases, PTC excluding PTMC were 210 cases. 143 patients underwent lateral lymph node dissection due to the positive preoperative biopsy. Lateral neck lymph node dissection was performed in 5 cases with high imaging suspicion but negative puncture results. 3 cases of them had lateral neck lymph node metastasis and 2 cases were confirmed negative during operation.

By comparing the positive and negative group, the results showed that males $\left(x^{2}=18.011, P<0.001\right)$, age $(t=9.132, P<0.001)$, age $<55$ years $\left(x^{2}=20.599, P<0.001\right)$, maximum tumor diameter $(t=-13.922, P<$ $0.001)$ and volume $(\mathrm{t}=-7.927, \mathrm{P}<0.001)$, cross-sectional aspect ratio $(\mathrm{t}=3.895, \mathrm{P}<0.001)$ and longitudinal aspect ratio $(t=5.721, P<0.001)$ were all significantly related to CLNM (Table 1$)$.

Table 1

Univariate analysis of correlative factors of CLNM in PTC

\begin{tabular}{|c|c|c|c|c|}
\hline Variable & $\begin{array}{l}\text { Positive group }(n= \\
\text { 432) }\end{array}$ & $\begin{array}{l}\text { Negative group }(n= \\
903)\end{array}$ & Statistics & $\mathbf{P}$ \\
\hline Gender (male/female) & $127 / 305$ & $172 / 731$ & $\begin{array}{l}x^{2}= \\
18.011\end{array}$ & $\begin{array}{l}P< \\
0.001\end{array}$ \\
\hline Age & $41.20 \pm 12.20$ & $47.30 \pm 11.02$ & $t=9.132$ & $\begin{array}{l}P< \\
0.001\end{array}$ \\
\hline Age $(\geq 55$ years/<55years $)$ & $61 / 371$ & $226 / 677$ & $\begin{array}{l}x^{2}= \\
20.599\end{array}$ & $\begin{array}{l}P< \\
0.001\end{array}$ \\
\hline $\begin{array}{l}\text { Tumour maximum } \\
\text { diameter(mm) }\end{array}$ & $14.4 \pm 10.0$ & $8.4 \pm 5.7$ & $t=-13.922$ & $\begin{array}{l}P< \\
0.001\end{array}$ \\
\hline Tumour volume(ml) & $2.1 \pm 4.6$ & $0.6 \pm 2.4$ & $t=-7.927$ & $\begin{array}{l}P< \\
0.001\end{array}$ \\
\hline Cross-sectional aspect ratio & $1.02 \pm 0.28$ & $1.08 \pm 0.26$ & $t=3.895$ & $\begin{array}{l}P< \\
0.001\end{array}$ \\
\hline Longitudinal aspect ratio & $0.88 \pm 0.29$ & $0.98 \pm 0.31$ & $t=5.721$ & $\begin{array}{l}P< \\
0.001\end{array}$ \\
\hline
\end{tabular}

We divided two sub-groups in positive group, there were PTMC and PTC excluding PTMC. Cross-sectional aspect ratio $\geq 1$ had significant difference for PTC patients excluding PTMC to predict CLNM (Table 2). 
Table 2

Univariate analysis of PTMC and PTC excluding PTMC in the positive group

\begin{tabular}{|llll|}
\hline Variable & $\begin{array}{l}\text { Cross- } \\
\text { sectional } \\
\text { aspect ratio } \geq\end{array}$ & $\begin{array}{l}\text { longitudinal section aspect ratio } \\
\mathbf{1}\end{array}$ & $P$ \\
\hline PTMC + CLNM (181) & 118 & 93 & $P=0.006$ \\
$\begin{array}{l}\text { PTC excluding PTMC + CLNM } \\
(251)\end{array}$ & 80 & 32 & \\
\hline
\end{tabular}

Gender, age, maximum tumor diameter and volume, cross-sectional and longitudinal aspect ratios were all included in the logistical analysis. The results showed that males $(\mathrm{OR}=1.723,95 \% \mathrm{Cl} 1.281-2.317, \mathrm{P}$ $<0.001)$, age $(\mathrm{OR}=0.960,95 \% \mathrm{Cl} 0.949-0.971, \mathrm{P}<0.001)$, maximum tumor diameter $(\mathrm{OR}=1.188,95 \% \mathrm{Cl}$ $1.148-1.230, \mathrm{P}<0.001)$ and volume $(\mathrm{OR}=0.838,95 \% \mathrm{Cl} 0.780-0.902, \mathrm{P}<0.001)$ were independent correlative factors for CLNM (Table 3).

Table 3

Logistic regression analysis of correlative factors of CLNM in PTC

\begin{tabular}{|llll|}
\hline Variable & OR & $95 \% \mathrm{Cl}$ & $\mathrm{P}$ \\
\hline Gender(male/female) & 1.723 & $1.281 \sim 2.317$ & $\mathrm{P}<0.001$ \\
\hline Age & 0.960 & $0.949 \sim 0.971$ & $\mathrm{P}<0.001$ \\
\hline Tumour maximum diameter & 1.188 & $1.148 \sim 1.230$ & $\mathrm{P}<0.001$ \\
\hline Tumour volume & 0.838 & $0.780 \sim 0.902$ & $\mathrm{P}<0.001$ \\
\hline Cross-sectional aspect ratio & 1.166 & $0.625 \sim 2.173$ & $\mathrm{P}=0.629$ \\
\hline Longitudinal aspect ratio & 1.313 & $0.751 \sim 2.298$ & $\mathrm{P}=0.340$ \\
\hline
\end{tabular}

Logistic regression analysis was carried out to determine independent correlative factors of CLNM. Gender, age, maximum tumor diameter and volume were analyzed using the ROC curve. The AUC, specificity, and sensitivity of the maximum tumor diameter was $0.738,66.6 \%$, and $69.9 \%$, respectively; the AUC, specificity, and sensitivity of the tumor volume was $0.733,67.6 \%$, and $68.1 \%$, respectively (Table 4 ). 
Table 4

AUC and cut-off values of correlative factors for CLNM in PTC

\begin{tabular}{|lllll|}
\hline Variable & AUC & Specificity & Sensitivity & Cut-off value \\
\hline Gender(male/female) & 0.552 & $81.0 \%$ & $29.4 \%$ & Not applicable \\
\hline Age & 0.355 & $99.9 \%$ & $0.2 \%$ & 80 \\
\hline Tumor maximum diameter & 0.738 & $66.6 \%$ & $69.9 \%$ & $8.4 \mathrm{~mm}$ \\
\hline Tumor volume & 0.733 & $67.6 \%$ & $68.1 \%$ & $0.2 \mathrm{ml}$ \\
\hline
\end{tabular}

According to the differences in clinical and ultrasonic characteristics using ANOVA, the patients were divided into three pair-to-pair comparison groups: only central metastasis group, only lateral metastasis group and both metastasis group. The results showed that the significant variable $(P<0.05)$ between the only central and only lateral metastasis groups, the only central and both metastasis group was maximum tumor diameter. The significant variables $(P<0.001)$ between the only central and both metastasis group included tumor volume, cross-sectional aspect ratio and longitudinal aspect ratio. The comparison between the three pair-to-pair groups is shown in Table 5.

Table 5

ANOVA variance analysis for CLNM in PTC

\begin{tabular}{|llll|}
\hline Variable & Central v.s. lateral & Central v.s. both & Lateral v.s. both \\
\hline Gender(male/female) & $\mathrm{P}=1.000$ & $\mathrm{P}=0.677$ & $\mathrm{P}=0.912$ \\
\hline Age & $\mathrm{P}=0.725$ & $\mathrm{P}=0.743$ & $\mathrm{P}=0.272$ \\
\hline Tumor maximum diameter & $\mathrm{P}=0.002^{\mathrm{a}}$ & $\mathrm{P}<0.001^{\mathrm{a}}$ & $\mathrm{P}=0.270$ \\
\hline Tumor volume & $\mathrm{P}=0.121$ & $\mathrm{P}<0.001^{\mathrm{a}}$ & $\mathrm{P}=0.987$ \\
\hline Cross-sectional aspect ratio & $\mathrm{P}=0.431$ & $\mathrm{P}<0.001^{\mathrm{a}}$ & $\mathrm{P}=0.768$ \\
\hline Longitudinal aspect ratio & $\mathrm{P}=0.124$ & $\mathrm{P}<0.001^{\mathrm{a}}$ & $\mathrm{P}=0.104$ \\
\hline a represented $\mathrm{P}<0.05$ & & & \\
\hline
\end{tabular}

\section{Discussion}

Thyroid carcinoma is a common endocrine malignant carcinoma, and PTC has the highest incidence of all thyroid carcinomas. PTC shows low malignant growth with a low incidence of distant metastasis and a low mortality rate; however, CLNM tends to occur at the early stage [14]. Lymph node metastasis in PTC is related to the diameter, location, number, and invasive growth of the primary tumor [14-15]. There is no uniform conclusion on the correlation between gender, age, pathological type and lymph node metastasis [16-17]. Ultrasonography is the primary examination method for the thyroid gland, but the detection of 
cervical lymph nodes by US is $18.8 \%-31 \%$, and is limited due to interference from the trachea, esophagus, osseous tissue, underlying thyroid diseases and the examiner's experience [18-19]. Therefore, evaluation of the correlative factors of CLNM in PTC has great clinical value. Previous similar studies have not provided consistent conclusions, as some included fewer cases, some included multifocality, and some included complex parameter characteristics. This study only enrolled unifocal PTC and analyzed the clinical information and US measurements. A stratified study is necessary, with additional subsequent and multiple ultrasonic signs, TI-RADS (Thyroid Imaging, Reporting and Data System), multifocality, pathological types and so on, in order to obtain more complete and systematic research results.

PTC is more common in female patients, with a male to female ratio of approximately $1: 3$, and the ratio in this study was 1: 3.46. Mao et al. [20] and Heaton et al. [21] reported that women and elderly patients were at risk of PTC, while men and younger patients were at risk of CLNM. Sun et al. [22] confirmed that men had an increased risk of central neck lymph node metastases. In this study, CLNM occurred in $42.5 \%$ $(127 / 299)$ of male patients, and occurred in only $29.4 \%$ (305/1036) of female patients, which also suggests that male patients have a higher risk of lymph node metastasis. This may be related to high hormone levels in male patients, which is consistent with results reported in the literature.

PTC occurs in all age ranges, with a high incidence between the age of 30 and 60 years. The mean age in the positive group included in this study was younger than that in the negative group, and the difference was statistically significant $(P<0.001)$, which was also consistent with previous reports where age was an independent risk factor for CLNM in PTC [23-24]. We used 55 years as the threshold in this study according to the TNM staging system for thyroid cancer [13], and $35.4 \%(371 / 1048)$ of patients who were younger than 55 years had CLNM, and among those who were 55 years or older, lymph node metastases occurred in only $21.2 \%(61 / 287)$ of patients in this study. This was consistent with the study reported by Zhou et al. [4], where age was an independent risk factor for CLNM, and the risk of CLNM in patients who were younger than 55 years was 2.6 times that of patients who were 55 years or older.

The maximum tumor diameter is an important reference index for PTC treatment protocols and the range of surgery [8]. The diameter of the lesion was closely related to invasion of the tumor. The growth of a malignant tumor is a process of self-proliferation and external invasion, the range of invasion continues to expand, and the contact area between the cancer focus and the capsule, blood vessels, and lymphatic vessels of the thyroid also increases [22], thereby the incidence of lymph node metastasis also increases [25]. A retrospective analysis based on large samples has shown that CLNM tended to occur in patients with a maximum tumor diameter of $10 \mathrm{~mm}$ or larger [26-27]. The results demonstrated that the maximum tumor diameter in the positive group was about 1.7 times that in the negative group. The tumor seemed to be ellipsoid, and the volume as the evaluation parameter made the result more objective and scientific. The tumor volume in the positive group was about 3.5 times that in the negative group. Tumor volume was significantly different between the only central metastasis group and the central and lateral metastasis group $(P<0.001)$. For larger tumors, cervical lymph nodes should be examined in order to improve the detection rate of CLNM. In particular, in patients with large volume tumors, central or both central and lateral lymph node metastasis should be determined in advance. If both of these 
examinations were added to routine thyroid and cervical lymph node ultrasonic screening, it would provide a guide for performing FNA for the detection of CLNM before surgery.

An aspect ratio $\geq 1$ is a highly specific index for the diagnosis of malignant thyroid nodules [28-29]. Zhan et al. [30] showed that the sensitivity of the aspect ratio in the differential diagnosis of benign and malignant thyroid nodules decreased gradually as the volume of thyroid nodules increased, and an aspect ratio $\geq 1$ was more significant for the diagnosis of PTC with a smaller volume. Nam et al. [31] divided 488 cases of PTC into two groups with one group having malignant ultrasonic signs, including an aspect ratio $>1$, solid mass with low echo, microcalcification, and a blurred boundary, while the other group had no malignant ultrasonic signs. A comparison between these two groups showed that patients with PTC and malignant signs were more prone to CLNM. Studies by Zhou et al. [4] showed that an aspect ratio > 1 in 1174 cases of unifocal PTC was a risk factor for CLNM. Deng et al. [32] reported that no statistical significance was seen in 908 PTC patients with an aspect ratio $\geq 1$ in predicting cervical lateral lymph node metastasis. Combined with previous literature, on the one hand, there was no distinction between the transverse or longitudinal aspect ratio; on the other hand, the correlation between the aspect ratio and PTC CLNM was inconsistent.

According to the morphology of the tumor, univariate analysis demonstrated that the cross-sectional aspect ratio and longitudinal-section aspect ratio were both statistically significant $(P<0.001)$ in this study, while logistic regression analysis showed that there was no statistically significant difference between the two groups. the main reason was that PTMC patients were $65.5 \%(874 / 1335)$ in the study, just $20.7 \%$ (181/874) were in the positive group. We compared cross-sectional and longitudinal section aspect ratio for PTMC and PTC patients excluding PTMC, it could be concluded that cross-sectional aspect ratio had a better predictive value for CLNM in PTC excluding PTMC, compared with the longitudinal aspect ratio. There was less relevant literature with the association between cross-sectional and longitudinal section aspect ratios in predicting cervical LN metastasis, especially for PTC patients excluding PTMC. We will collect more data for further research in the future.

The limitations of this study were as follows: 1 . This was a single-center retrospective study including unifocal PTC and lymph node dissection performed in the central area,which may have introduced subjective bias. 2. Cases with metastases in lateral location were not adequate, large samples are required to study the cervical metastases in different parts. For skip lateral lymph node metastases, more effective preoperative assessment should be adopted. 3 . This is the preliminary study for large sample size of PTC patients, we will add detailed TI-RADS, clinical and pathological staging, subdivided pathological types, machine learning models for further research.

\section{Conclusions}

In summary, based on PTC pathology, the correlations between gender, age, US measurements and CLNM were analyzed in order to assess the risk of lymph node metastasis before surgery. For PTC patients with risk factors such as younger age, male gender and larger tumor, more detailed preoperative lymph node 
examination should be conducted, including a cervical lymph node sonographic scan by experienced sonologists (with more than 10 years' experience) and enhanced CT scan of the neck as a rule, which will cover a wide range of thyroid cancer population and help develop a more reasonable clinical treatment protocol.

\section{Abbreviations}

PTC Papillary thyroid carcinoma

CLNM Cervical lymph node metastasis

PTMC Papillary thyroid microcarcinoma

FNA Fine-needle aspiration biopsy

US Ultrasonography

ROC Receiver operating characteristic

OR Odds ratio

$\mathrm{Cl}$ Confidence interval

AUC Area under the curve

\section{Declarations}

\section{Ethics approval and consent to participate}

This study was approved by the Ethics Committee of the Cancer Hospital of the University of Chinese Academy of Sciences (Zhejiang Cancer Hospital). All methods were carried out in accordance with relevant guidelines and regulations. Written Informed consent was obtained from all patients, and it was obtained from a parent or guardian for participants under 16 years old.

\section{Consent for publication}

The scientific guarantor of this publication is Prof. Dong Xu. The permission has been received that any material in the article can be used, and consent for publication is acquired from all authors.

\section{Availability of data and materials}

The datasets used and analysed during the study are available from the corresponding author on reasonable request. 
We define that all authors haven't involved in a set of conditions in which our professional judgment concerning the validity of research, and we aren't influenced by financial gain. Data and material are available and reliable in this article.

\section{Funding}

This study was supported by National Natural Science Foundation of China (NO. 82071946) and Zhejiang Provincial Natural Science Foundation of China (NO. LSD19H180001). DX is responsible for these two funds.

\section{Authors' contributions}

DX had full access to all the data and took responsibility for the integrity and accuracy of the data analysis. LHZ and JCY were major contributors in writing the manuscript. QDZ participated in collection and management of the data. YC contributed to analyse and interpret the data. All authors read and approved the final manuscript.

\section{Acknowledgement}

This manuscript has been released as a pre-print at Research Square, DOI:10.21203/rs.3.rs-21257/v1[31].

\section{References}

1. Lim H, Devesa SS, Sosa JA, et al. Trends in thyroid cancer incidence and mortality in the united states, 1974-2013[J]. JAMA, 2017, 317(13):1338-1348. DOI:10.1001/jama.2017.2719.

2. Chen W, Zheng R, Baade PD, et al. Cancer statistics in China, 2015[J]. CA Cancer J Clin, 2016, 66(2):115-132. DOI:10.3322/caac.21338.

3. Kim SY, Kwak JY, Kim EK, et al. Association of preoperative US features and recurrence in patients with classic papillary thyroid carcinoma[J]. Radiology, 2015, 277(2):574-583.

DOI:10.1148/radiol.2015142470.

4. Zhou J, Zhou SC, Li JW, et al. Risk factors of central neck lymph node metastasis following solitary papillary thyroid carcinoma[J].Chin J Ultrasonogr,2019, 28(3):235-240.

5. Li JW, Chang C, Chen M, et al. Identification of calcifications in thyroid nodules: comparison between ultrasonography and CT[J]. Chin J Ultrasonogr.2017; 26(5):313317. DOI:10.3760/cma.j.issn.1004-4477.2017.05.012.

6. Zhu Q, Li JW, Zhou SC, Chang C, Chen M, Fan YW. Diagnostic value of ultrasonography in predicting neck lymph node metastasis in Hashimoto's thyroiditis with papillary thyroid carcinoma[J]. Chin J Ultrasonogr. 2016; 25(11):962-965. DOl:10.3760/cma.j.issn.1004-4477.2016.11.011.

7. Sun $\mathrm{W}$, Lan $\mathrm{X}$, Zhang $\mathrm{H}$, et al. Risk factors for central lymph node metastasis in CNO papillary thyroid carcinoma: a systematic review and meta-analysis[J]. PLos One, 2015, 10(10): e0139021.

DOI:10.1371/journal. pone, 0139021. 
8. Xiang $D$, Xie $L, X u Y$, et al. Papillary thyroid microcarcinomas located at the middle part of the middle thyroid of the thyroid gland correlates with the presence of neck metastasis[J]. Surgery, 2015, 157(3):526-533. DOI:10.1016/j.surg.2014.10.020.

9. Tao Y, Wang C, Li L, et al. Clinicopathological features for predicting central and lateral lymph node metastasis in papillary thyroid microcarcinoma: Analysis of 66 cases that underwent central and lateral lymph node dissection[J]. Mol Clin Oncol, 2017, 6(1):49-55. DOI:10.3892/mco.2016.1085.

10. Chinese Association of Thyroid Oncology (CATO). Chinese experts' consensus on diagnosis and treatment of thyroid micropapillary carcinoma (2016 Edition). Chin J Clin Oncol. 2016;43(10):405411.

11. Chinese Thyroid Association (CTA), Thyroid Committee of Chinese Research Hospital Association. Expert consensus on lymph node dissection in cervical region of differentiated thyroid cancer (2017 Edition) [J]. Chin J Practical Surg. 2017;37(9):985-991.

12. Dai WJ, Yu QA. Some technics of central lymph node dissection of thyroid cancer[J]. J Clin Surg, 2017, 25(11):826-828.

13. Haugen BR, Alexander EK, Bible KC, et al. 2015 American Thyroid Association Management Guidelines for Adult Patients with Thyroid Nodules and Differentiated Thyroid Cancer: The American Thyroid Association Guidelines Task Force on Thyroid Nodules and Differentiated Thyroid Cancer[J], 2016, 26(1):1-133. DOI:10.1089/thy.2015.0020.

14. Wang TS, Evans DB. Commentary on: Occult lymph node metastasis and risk of regional recurrence in papillary thyroid cancer after bilateral prophylactic central neck dissection: A multi-institutional study[J]. Surgery, 2017, 161(2):472-474.

15. Park JP, Roh JL, Lee JH, et al. Risk factors for central neck lymph node metastasis of clinically noninvasive, node-negative papillary thyroid microcarcinoma[J]. Am J Surg, 2014, 208(3):412-418. DOI:10.1016/j. amjsurg. 2013.10.032.

16. AL AA, Williams BA, Rigby MH, et al. Multifocal papillary thyroid cancer increases the risk of central lymph node metastasis[J]. Thyroid, 2015, 25(9):1008-1012. DOI:10.1089/thy. 2015.0130.

17. Lin DZ, Qu N, Shi RL, et al. Risk prediction and clinical model building for lymph node metastasis in papillary thyroid microcarcinoma[J]. Onco Targets Ther, 2016, 9:5307-5316.

DOI:10.2147/OTT.S107913.

18. Qu N, Zhang L, Lin DZ, et al. The impact of coexistent Hashimoto's thyroiditis on lymph node metastasis and prognosis in papillary thyroid microcarcinoma[J]. Tumour Biol, 2016, 37(6):76857692. DOI:10.1007/s13277-015-4534-4.

19. Li JW, Chang C, Chen M, et al. Identification of calcifications in thyroid nodules: comparison between ultrasonography and CT[J]. Chin J Ultrasonogr,2017, 26(5):313317. DOI:10.3760/cma.j.issn.1004-4477.2017.05.012.

20. Mao LN, Wang P, Li ZY, et al. Risk factor analysis for central nodal metastasis in papillary thyroid carcinoma[J]. Oncol Lett, 2015, 9(1):103-107. 
21. Heaton CM, Chang JL, Orloff LA. Prognostic implications of lymph node yield in central and lateral neck dissections for well-differentiated papillary thyroid carcinoma[J]. Thyroid, 2016,26(3):434-440.

22. Sun YS, Lv HJ, Zhao YR, et al. Risk factors for central neck lymph node metastases of papillary thyroid carcinoma[J]. Chin J Otorhinolaryngol Head Neck Surg, 2017, 52(6):421-425.

23. Zhu Q, Li JW, Zhou SC, et al. Diagnostic value of ultrasonography in predicting neck lymph node metastasis in Hashimoto's thyroiditis with papillary thyroid carcinoma[J]. Chin J Ultrasonogr,2016, 25(11):962-965. DOI:10.3760/cma.j.issn.1004-4477.2016.11.011.

24. Ma B, Wang Y, Yang S, et al. Predictive factors for central lymph node metastasis in patients with cN0 papillary thyroid carcinoma: A systematic review and meta-analysis[J]. Int J Surg, 2016, 28:153161.

25. Lan Y, Song Q, Jin Z, et al. Correction of routine ultrasound features and BRAFV600E gene to the cervical lymph node metastasis of thyroid papillary carcinoma[J]. Med J Chin PLA, 2019, 44(9):747752.

26. Jiang LH, Chen C, Tan Z, et al. Clinical characteristics related to central lymph node metastasis in cN0 papillary thyroid carcinoma: a retrospective study of 916 patients[J]. Int J Endocr, 2014, 2014:385787.

27. Wang XQ, Wei W, Wei $X$, et al. Study on the relationship between ultrasonographic features of papillary thyroid carcinoma and central cervical lymph node metastasis[J]. Chin J Oncol, 2018, 40(3):196-200.

28. Kwak JY, Han $\mathrm{KH}$, Yoon JH, et al. Thyroid imaging reporting and data system for US features of nodules: a step in establishing better stratification of cancer risk. Radiology, 2011;260:892-899.

29. Na DG, Baek JH, Sung JY, et al. Thyroid imaging reporting and data system risk stratification of thyroid nodules: categorization based on solidity and echogenicity. Thyroid, 2016, 26:562-572.

30. Zhan WW, Xu SY. New progress of thyroid nodule ultrasonography[J/CD]. Chin J Med Ultrasound (Electronic Edition), 2013, 10(2):88-93.

31. Nam SY, Shin JH, Han BK, et al. Preoperative ultrasonographic features of papillary thyroid carcinoma predict biological behavior[J]. J Clin Endocrinol Metab, 2013, 98(4):1476-1482.

32. Deng SP, Xiong HH, Li QS, et al. Ultrasonographic characteristics of papillary thyroid cancer: predicting factors of lateral lymph node metastasis[J]. Chinese J Ultrasound Med, 2017, 33(3):196198.

33. Zhou LH, Zhu QD, Yao JC, et al. Correlation analysis of nodular sonographic parameters with cervical lymph node metastasis in papillary thyroid carcinoma. 2021. DOI:10.21203/rs.3.rs-21257/v1[31]. 